\section{Migräneprophylaxe bei Kindern nicht besser als Placebo}

In der Prophylaxe von Migräneepisoden bei Kindern sind Amitriptylin und Topiramat nicht wirksamer als Placebo. In den USA wurde eine randomisierte Studie vorzeitig gestoppt, weil sich keinerlei Zusatznutzen abzeichnete. Die Therapie war von teilweise erheblichen Nebenwirkungen begleitet.

D ass die in der Erwachsenenmedizin zur Migräneprophylaxe zugelassenen Medikamente Amitriptylin und Topiramat bei Kindern offenbar wirkungslos sind, ist das ernüchternde Resultat einer Studie, die von der USGesundheitsbehörde National Institutes of Health unterstützt wurde. „Das Modell der Kopfschmerzbehandlung, welches bei Erwachsenen funktioniert, scheint sich nicht auf Kinder übertragen zu lassen", schreiben die Studienautoren um Scott W. Powers vom University of Cincinnati College of Medicine.

In Deutschland gehört das Antikonvulsivum Topiramat in der Migräneprophylaxe bei Erwachsenen zur ersten Wahl, wohingegen Amitriptylin, ein trizyklisches Antidepressivum, lediglich als zweite Wahl empfohlen wird. Für Kinder sind beide Medikamente zumindest hierzulande nicht zugelassen.

An der randomisierten Studie, die an 31 US-Zentren lief, haben 361 Kinder und Jugendliche im Alter zwischen 8 und 17 Jahren mit der Diagnose Migräne gemäß den Kriterien der International Classification of Headache Disorders teilgenommen. Alle Teilnehmer wurden zunächst gebeten (ggf. mit Hilfe ihrer Eltern) einen
Monat lang Tagebuch über das Auftreten von Kopfschmerzen zu führen.

Die Zahl der Kopfschmerztage vor Beginn der medikamentösen Therapie lag demnach bei durchschnittlich 11,4 Tagen. Anschließend wurden die Patienten im Verhältnis 2:2:1 einer von drei Gruppen zugelost: Die erste erhielt Amitriptylin, die zweite Topiramat und die dritte Placebo in gleich aussehenden Kapseln, jeweils zweimal täglich. Diese Therapie sollte über 24 Wochen fortgesetzt werden, wobei auf eine achtwöchige Einschleichphase eine 16-wöchige Erhaltungsphase folgte. Primärer Endpunkt der Studie war die mindestens $50 \%$ ige Reduktion der Kopfschmerztage im letzten Studienmonat gegenüber dem Monat vor Therapiebeginn.

Schon die erste Interimsanalyse förderte jedoch klar den fehlenden Zusatznutzen der Medikamente gegenüber Placebo zutage: Mit einer bedingten Teststärke von $16 \%$ von Amitriptylin gegenüber Placebo und von $14 \%$ für Topiramat versus Placebo wurde die vorab festgesetzte Effektivitätsschwelle gegenüber dem Scheinmedikament - sie lag bei $20 \%$ - in beiden Fällen deutlich unterschritten. Auf Anraten des

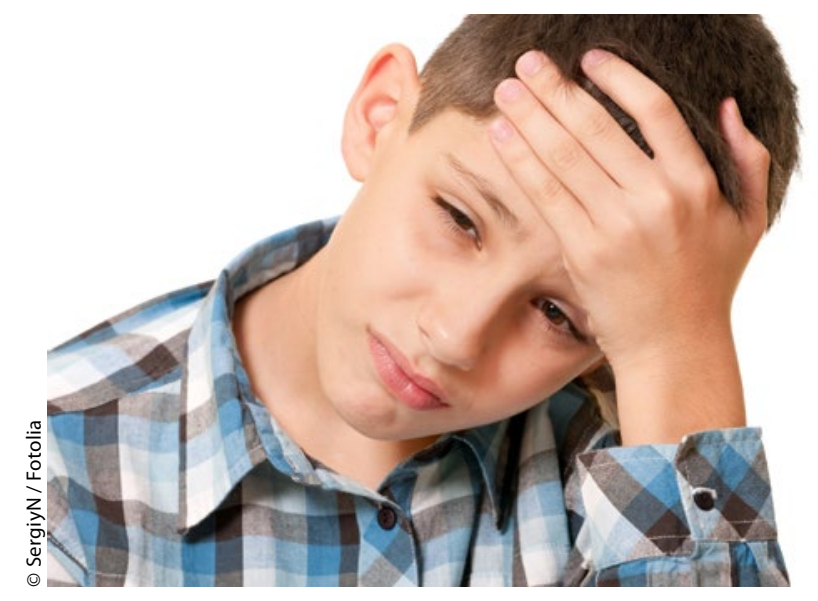

Die gängige Mirgräneprophylaxe bei Erwachsenen scheint bei Heranwachsenden nicht zum Erfolg zu führen.
Sicherheitsüberwachungsausschusses wurde die Studie daraufhin gestoppt.

Den primären Endpunkt erreicht hatten $52 \%$ in der Amitriptylin-, $55 \%$ in der Topiramat- und $61 \%$ in der PlaceboGruppe. Die Unterschiede, sowohl des jeweiligen Medikaments gegenüber Placebo als auch der Medikamente untereinander, waren auch nach Herausrechnen von Altersunterschieden und Unterschieden in der ursprünglichen Zahl der Kopfschmerztage nicht signifikant. Gegenüber dem Ausgangszeitraum hatte sich die Zahl der Kopfschmerztage, bezogen auf den letzten Studienmonat, unter Amitriptylin und Topiramat um jeweils 6,7 Tage verringert, unter Placebo um 5,9 Tage.

Der Einsatz der Medikamente hatte den Kindern offenbar nicht nur nicht genutzt: Im Vergleich zu Placebo traten in der Amitriptylin-Gruppe deutlich häufiger Fatigue und Mundtrockenheit auf, in der Topiramat-Gruppe kam es signifikant häufiger zu Parästhesien und Gewichtsabnahme. Insgesamt zwölf Patienten entwickelten schwere Nebenwirkungen: Unter Amitriptylin kam es in drei Fällen zu Stimmungsschwankungen, in einem Fall trat eine Synkope auf. Ein mit Topiramat behandelter Patient versuchte, sich das Leben zu nehmen. Allerdings zeigten sich im Child Depression Inventory keine nennenswerten Unterschiede zwischen den Gruppen.

Fazit: Die Bilanz der Studie fällt alles in allem negativ aus: „Die Daten“, so Powers, „zeigen für keine der beiden Substanzen einen Vorteil im Nutzen-RisikoProfil“; dies gelte zumindest für die untersuchte Spanne von zwei Monaten. Erst kürzlich hat die US-Behörde FDA Topiramat für Heranwachsende im Alter zwischen 12 und 17 Jahren zugelassen. Nach Powers und Kollegen wird man sich jedoch in der Migräneprophylaxe bei Kindern möglicherweise umorientieren müssen. Vor allem die in der Studie nachgewiesene hohe Ansprechrate auf Placebo sei etwas, das man vielleicht für zukünftige Strategien nutzen könnte.

Dr. Elke Oberhofer

Powers SW et al. Trial of Amitriptyline, Topiramate, and Placebo for Pediatric Migraine. N Engl J Med 2017;376(2):115-24. 